\title{
Fractional flow reserve and intravascular scan as part of the coronary fistulas diagnostic process: Future perspectives
}

\author{
Martino Pepe ${ }^{1}$, Francesco Giardinelli ${ }^{1}$, Alessandro Cafaro ${ }^{2}$, Filippo Masi ${ }^{1}$, \\ Eliano Pio Navarese ${ }^{3}$, Antonio Tito ${ }^{1}$, Fabrizio Resta ${ }^{4}$, \\ Annagrazia Cecere ${ }^{1}$, Marco Matteo Ciccone ${ }^{1}$, Stefano Favale ${ }^{1}$ \\ ${ }^{1}$ Cardiovascular Diseases Section, Department of Emergency and Organ Transplantation (DETO), \\ University of Bari, Bari, Italy \\ ${ }^{2}$ Department of Cardiology, General Hospital "F. Miulli”, Acquaviva delle Fonti, Bari, Italy \\ ${ }^{3}$ Department of Internal Medicine, Division of Cardiology, Pulmonology and Vascular Medicine, \\ Heinrich-Heine-University, Düsseldorf, Germany \\ ${ }^{4}$ Department of Cardiology, Hospital "Santa Maria”, GVM Care and Research, Bari, Italy
}

A man aged 74 presented to the Emergency Room with ST-elevation in anterior leads (Fig. 1A, B). An urgent coronary angiography revealed the absence of significant coronary stenoses, but did reveal multiple coronary artery fistulas apparently draining into the pulmonary artery (Fig. $1 \mathrm{C}-\mathrm{F}$ ); at the level of left anterior descending (LAD) angiography showed a dilated proximal tract, and, downstream from the fistula, a reduced and intermittent flow. Coronary computed tomography angiography confirmed multiple and complex arteriovenous malformations. The patient underwent further invasive evaluations which included a pulmonary to systemic blood flow ratio $(\mathrm{Qp} / \mathrm{Qs})$ calculation, intravascular ultrasound scan (IVUS) and fractional flow reserve (FFR) on LAD evaluation. The right heart catheterization detected high pulmonary artery pressure $(48 / 18 \mathrm{mmHg})$ and modest left-to-right shunt (Qp/Qs 1.08). IVUS detected no significant atherosclerotic disease on LAD, an augmented caliber proximally to the fistula with a diameter ranging from 6 to $7 \mathrm{~mm}$, and a unique fistula localized at the end of the first tract (Fig. 1G-J). FFR distally to the fistula ostium was 0.82 (slightly above the most used cut-off for a treatment decision in cases of coronary stenoses), and during the pullback a pressure jump was evident across the fistula with an FFR value in the proximal LAD of 0.92 . No interventions were scheduled and the patient was discharged on medical therapy; at 24 months the patient kept asymptomatic and no adverse events occurred.

The authors opinion is that this report raises cues about possible new diagnostic approaches which enhances the drive to future therapeutic choices in fistulas treatment.

Conflict of interest: None declared

Address for correspondence: Annagrazia Cecere, MD, Cardiovascular Diseases Section, Department of Emergency and Organ Transplantation (DETO), University of Bari, Italy, tel: 0039 0805592996, e-mail: agcecere@gmail.com Received: 15.01.2018 Accepted: 17.01.2018 


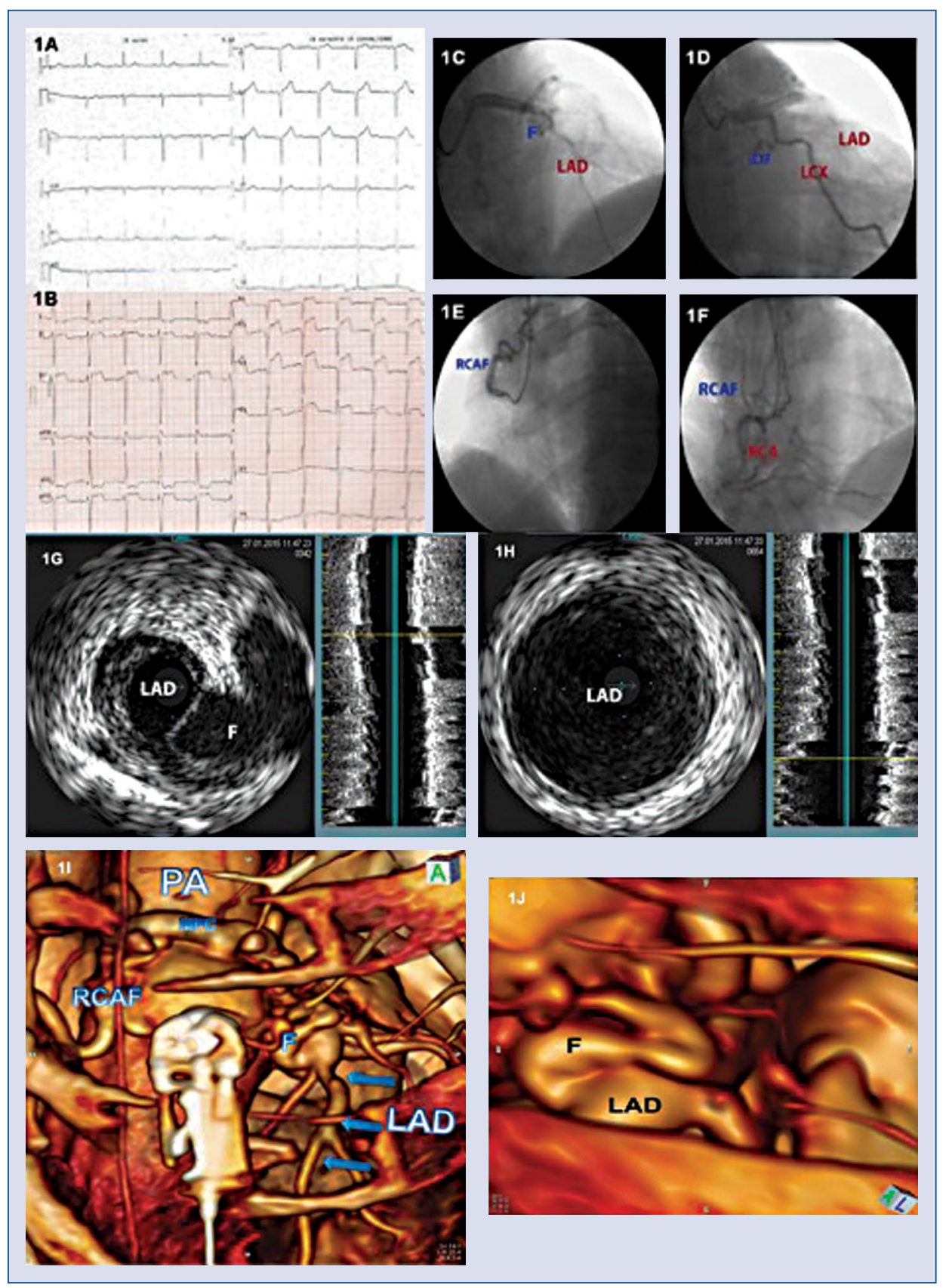

Figure 1. A. Previous electrocardiogram; B. Electrocardiogram at admission showing anterior ST-elevation. C, D. Coronary angiography: multiple fistulas emerging from all the epicardial vessels with apparent drainage into the pulmonary artery; C. At left anterior descending coronary artery (LAD) level is evident the proximal ectatic tract, the origin of the fistula, and a barely visible medium-distal segment (suggesting medium of contrast stealing); D. A fistula emerging from left circumflex coronary artery (LCX); E, F. Selective and non-selective opacification of fistulas emerging from the right coronary artery (RCA). G, H, I, J. Intravascular ultrasound scan (IVUS) and corresponding coronary tomography (CT) views: G. IVUS view at the ostium of LAD fistula; H. IVUS image proximally to LAD fistula's origin, showing no significant atherosclerosis and ectasia; I, J. CT multiplanar three-dimensional reconstruction of LAD fistula: an anomalous vessel originating with a single crooked ostium from the proximal LAD posterior wall and draining into the main collector of the pulmonary artery; AO — aorta; F — fistula; FC — fistulas collectors; MFC — main fistulas collector; PA - pulmonary artery. 